\title{
Solution to the Riemann problem for a five-equation model of multiphase flows in non-conservative form
}

\author{
SAHADEB KUILA $^{1}$, T RAJA SEKHAR ${ }^{2, *}$ and G C SHIT $^{1}$ \\ ${ }^{1}$ Department of Mathematics, Jadavpur University, Kolkata 700032, India \\ ${ }^{2}$ Department of Mathematics, Indian Institute of Technology Kharagpur, Kharagpur 721302, India \\ e-mail: trajasekhar@maths.iitkgp.ernet.in; gcs@math.jdvu.ac.in
}

MS received 3 September 2015; revised 5 April 2016; accepted 2 May 2016

\begin{abstract}
In this paper, we consider the Riemann problem for a five-equation, two-pressure (5E2P) model proposed by Ransom and Hicks for an isentropic compressible gas-liquid two-phase flows. The model is given by a strictly hyperbolic, non-conservative system of five partial differential equations (PDEs). We investigate the structure of the Riemann problem and construct an approximate solution for it. We solve the Riemann problem for this model approximately assuming that all waves corresponding to the genuinely nonlinear characteristic fields are rarefaction and discuss their properties. To verify the solver, a series of test problems selected from the literature are presented.
\end{abstract}

Keywords. Two-phase flows; non-conservative model; Riemann problem; four-rarefaction approximation.

\section{Introduction}

In the recent past, considerable research has been devoted to the modeling and simulation of compressible two phase flows. Over the years various approaches have been proposed to develop mathematical models. Theoretical and numerical modeling of gas-liquid two-phase fluid flows are of practical importance in many areas of industry and have remained complex and challenging fields for mathematical research. The modeling and simulation of these flows is one of the most challenging problems in computational fluid dynamics. There are several two-phase flow models in the literature [1-7]. There is an important issue regarding these models that the models are either conservative or nonconservative form, that is to say, whether the basic equations have, or not, a known conservation-law form in the mathematical sense. Almost all the models in the literature have non-conservative form due to the interface interactions. Since these models contain non-conservative products and therefore, Rankine-Hugoniot relations cannot be defined in an unambiguous manner. In particular, an additional closure relation is needed to provide a full set of jump conditions.

For the solution of compressible two-fluid models, one can use exact Riemann solver or approximate Riemann solver. Andrianov and Warnecke [8] were the first to propose an inverse solution to the Riemann problem in the sense that the initial left and right states are obtained as a function of the intermediate states of the solution. On the

*For correspondence contrary, a direct iterative approach is used in Schwendema et al [9] leading to exact solutions of the Riemann problem for any initial left and right states. Deledicque and Papalexandris [10] presented an exact Riemann solver for compressible two-phase flow models containing non-conservative products. Another direct approach to construct theoretical solution is proposed by Castro and Toro [11] of the Riemann problem for the five-equation two-phase nonconservative model of Saurel and Abgrall [5]. Murrone and Guillard [12] have been studied an Eulerian diffuse interface model of Baer and Nunziato [13] type for the simulation of compressible multi-fluid and two-phase flow problems. We refer [14] for existence of weak solutions to the Riemann problem for the conservative hyperbolic systems. Thanh [15] introduced a phase decomposition approach to deal with the generalized Rankine-Hugoniot relations and then solved the Riemann problem for a model of two-phase flows. The Riemann problem for the widely used drift-flux compressible two-phase flow model have been studied by Kuila et al [16, 17]. A diffuse-interface method is proposed by Allaire [18] for the simulation of interfaces between compressible fluids with general equations of state, including tabulated laws. Radha and Sharma [20] discussed interaction of a weak discontinuity with elementary waves of Riemann problem for the Euler equations. Solution to the Riemann problem in a one-dimensional magnetogasdynamic flow have been discussed by Raja Sekhar and Sharma [19]. Kuila and Raja Sekhar $[21,22]$ studied the Riemann problem for quasilinear hyperbolic system of PDEs governing the one dimensional 
ideal and non-ideal isentropic magnetogasdynamics with transverse magnetic field.

In this work, a non-conservative five equation compressible two-phase model of Ransom and Hicks [3] is considered. The mathematical model comprises two-pressure of an isentropic equation of state. The two-phases are made of namely the gas phase and the liquid phase. Furthermore, there has not been an exact Riemann solver in the case of isentropic two-fluid models yet. So, one has to look for an approximate Riemann solver for the numerical solution of these types of two-phase models [3]. Ansari and Ghiasi [23] considered this model and investigated the hydro dynamical instability initiation criterion in two-phase air-water stratified flow using spectral method in a horizontal duct. The two-rarefaction approximation in single-phase gas dynamics was introduced by Toro [24]. In our study, we extend this approach to solve the non-conservative hyperbolic model, pioneered by Ransom and Hicks [3]. Mathematically, shock waves and associated Rankine-Hugoniot jump conditions cannot be defined for non-conservative form of original system. Because of this we made the assumption that all the genuinely nonlinear characteristic fields are associated with rarefaction waves. This is one of the most popular non-conservative models formulated by Ransom and Hicks [3]. But analytical and numerical solution is not available for this model in the literature. In this point of view, our theoretical solution by this approach is one of the significant works. If all genuinely nonlinear waves are in fact rarefaction, then the obtained solution is exact. Otherwise, our solution will be an approximation. We introduce the Riemann problem and study the characteristic analysis of the system. On this approximation, finally, we obtain a system of nonlinear algebraic equations and we solve them using Newton-Raphson iterative procedure with a stopping criteria where the relative error is less than $10^{-8}$; the initial guess for the intermediate unknown physical quantity is taken to be the average of left and right state.

This paper is structured as follows. In section 2, the fiveequation two-pressure model is proposed, and its conservative and primitive variable formation are analyzed. The Riemann problem and its characteristic framework is briefly discussed in section 3 . In section 4 , we assume that all genuinely nonlinear characteristic fields are associated with rarefaction waves and discuss the properties of the rarefaction waves. In section 5, we present the solution strategy of the Riemann problem and solvability of system of nonlinear algebraic equations. Numerical tests to illustrate the different choices are presented in section 6 . Section 7 is devoted to some concluding remarks.

\section{Basic equations}

We consider the two-phase flow model of Ransom and Hicks [3] that governs the dynamics of two compressible fluids (called phases, hereafter), such as gas and liquid mixture, by assuming that the flow is isentropic in both phases.

\subsection{Non-conservative model for two-phase flow}

The five-equation, two-pressure (5E2P) model which can be written in the non-conservative form as [23]:

$$
\begin{gathered}
\frac{\partial}{\partial t}\left(\alpha_{1} \rho_{1}\right)+\frac{\partial}{\partial x}\left(\alpha_{1} \rho_{1} u_{1}\right)=0 \\
\frac{\partial}{\partial t}\left(\alpha_{2} \rho_{2}\right)+\frac{\partial}{\partial x}\left(\alpha_{2} \rho_{2} u_{2}\right)=0 \\
\frac{\partial}{\partial t}\left(\alpha_{1} \rho_{1} u_{1}\right)+\frac{\partial}{\partial x}\left(\alpha_{1} \rho_{1} u_{1}^{2}\right)+\alpha_{1} \frac{\partial p_{1}}{\partial x}+\left(p_{1}-\tilde{p}\right) \frac{\partial \alpha_{1}}{\partial x}=0, \\
\frac{\partial}{\partial t}\left(\alpha_{2} \rho_{2} u_{2}\right)+\frac{\partial}{\partial x}\left(\alpha_{2} \rho_{2} u_{2}^{2}\right)+\alpha_{2} \frac{\partial p_{2}}{\partial x}+\left(p_{2}-\tilde{p}\right) \frac{\partial \alpha_{2}}{\partial x}=0, \\
\frac{\partial \alpha_{1}}{\partial t}+\tilde{u} \frac{\partial \alpha_{1}}{\partial x}=S .
\end{gathered}
$$

Index 1 in the above system is referred to the liquid phase and 2 to the gas phase; $x$ is the space coordinate and $t$ is the time; $\rho_{i}, u_{i}, p_{i}$ and $\alpha_{i}$ are the density, velocity, pressure and volume fraction of phase $i(i=1,2)$, respectively. The volume fractions are subject to the constraint

$$
\alpha_{1}+\alpha_{2}=1
$$

and $S$ is the source term on the right hand side of Eq. (5). Also, $\tilde{p}$ and $\tilde{u}$ denote the interface pressure and horizontal velocity which are respectively given by

$$
\tilde{p}=\left(a_{1} p_{2}+a_{2} p_{1}\right) /\left(a_{1}+a_{2}\right)
$$

and

$$
\tilde{u}=\left(u_{1}+u_{2}\right) / 2,
$$

where $a_{i}=c_{i} \rho_{i}$ is the acoustic impedance and $c_{i}^{2}=d p_{i} / d \rho_{i}$ is the isentropic sound speed of material $i(i=1,2)$. In particular, isentropic laws are considered in the following form

$$
p_{i}=p_{i}\left(\rho_{i}\right), \quad i=1,2 .
$$

Thus, the equation of state for a pure liquid and an ideal gas is written as follows

$$
p_{1}=p_{1}\left(\rho_{1}\right)=K_{1} \rho_{1}^{\gamma_{1}} \quad \text { and } \quad p_{2}=p_{2}\left(\rho_{2}\right)=K_{2} \rho_{2}^{\gamma_{2}}
$$

where $K_{1}, K_{2}, \gamma_{1}$ and $\gamma_{2}$ are constants to be specified.

Equations (1)-(5) form a first-order, quasi-linear and non-conservative system of PDEs for isentropic gas-liquid two-phase flow.

\subsection{Conservative and primitive-variables formulation}

To further establish the necessary mathematical framework of the current model equations for the development of a Riemann solver, we write the basic equations in the 
following form of conservative and primitive variables, respectively

$$
U=\left[\alpha_{2} \rho_{2}, \alpha_{2} \rho_{2} u_{2}, \alpha_{1} \rho_{1}, \alpha_{1} \rho_{1} u_{1}, \alpha_{1}\right]^{t r},
$$

and

$$
V=\left[\rho_{2}, u_{2}, \rho_{1}, u_{1}, \alpha_{1}\right]^{t r},
$$

where $t r$ denotes the transposition. The two-phase model derived by Ransom and Hicks [3] in the quasi-linear form with the conservative variable $U$ as

$$
\frac{\partial U}{\partial t}+M(U) \frac{\partial U}{\partial x}=0
$$

and the coefficient matrix $M(U)$ is given by

$$
\left(\begin{array}{ccccc}
0 & 1 & 0 & 0 & 0 \\
c_{2}^{2}-u_{2}^{2} & 2 u_{2} & 0 & 0 & -\left(1-\gamma_{2}\right) p_{2}+\tilde{p} \\
0 & 0 & 0 & 1 & 0 \\
0 & 0 & c_{1}^{2}-u_{1}^{2} & 2 u_{1} & \left(1-\gamma_{1}\right) p_{1}-\tilde{p} \\
0 & 0 & 0 & 0 & \tilde{u}
\end{array}\right)
$$

To develop wave analysis of the Riemann problem solution, we rewrite system of Eqs. (1)-(5) in non-conservative form with the primitive variable $V$ as

$$
\frac{\partial V}{\partial t}+M(V) \frac{\partial V}{\partial x}=0
$$

and the Jacobian matrix $M(V)$ is given by

$$
\left(\begin{array}{ccccc}
u_{2} & \rho_{2} & 0 & 0 & -\frac{\rho_{2}\left(u_{2}-\tilde{u}\right)}{\alpha_{2}} \\
\frac{c_{2}^{2}}{\rho_{2}} & u_{2} & 0 & 0 & -\frac{\left(p_{2}-\tilde{p}\right)}{\alpha_{2} \rho_{2}} \\
0 & 0 & u_{1} & \rho_{1} & \frac{\rho_{1}\left(u_{1}-\tilde{u}\right)}{\alpha_{1}} \\
0 & 0 & \frac{c_{1}^{2}}{\rho_{1}} & u_{1} & \frac{\left(p_{1}-\tilde{p}\right)}{\alpha_{1} \rho_{1}} \\
0 & 0 & 0 & 0 & \tilde{u}
\end{array}\right) .
$$

\section{The Riemann problem and some mathematical properties}

In this section, we define the Riemann problem

$$
\left.\begin{array}{c}
\frac{\partial V}{\partial t}+M(V) \frac{\partial V}{\partial x}=0, \\
V(x, 0)=\begin{array}{c}
V_{L}, x<0 \\
V_{R}, x>0
\end{array}
\end{array}\right\}
$$

where $V$ is the primitive vector of unknowns (9) and the Jacobian matrix $M(V)$ is given by (13). In the above, the states $V_{L}$ and $V_{R}$ represent, respectively, the left and right initial data of the Riemann problem (14).

\subsection{Characteristic analysis of the Riemann problem}

We derive the eigenvalues for both the systems (10) and (12). For both the matrices $M(U)$ and $M(V)$, eigenvalues are same and the five eigenvalues are

$$
\lambda_{1}=u_{1}-c_{1}, \lambda_{2}=u_{2}-c_{2}, \lambda_{3}=\tilde{u}, \lambda_{4}=u_{2}+c_{2}, \lambda_{5}=u_{1}+c_{1} .
$$

For all the physically acceptable states of the material the $u_{i}$ and $c_{i}$ are real numbers. Therefore, the model 5E2P has all real and distinct characteristic values for all physically acceptable states, so it is strictly hyperbolic as $c_{i}>0$ for $i=1,2$. The system is not strictly hyperbolic at vacuum as well as for zero speed. In our paper, we deal with the strictly hyperbolic problem by assuming that $\rho_{1}>0$ and $\rho_{2}>0$ for liquid and gas phase respectively. Furthermore, the corresponding right eigenvectors, for the conservativevariable formulation (10) follow by straight forward computation, are

$$
\begin{aligned}
R^{(1)}= & \left(\begin{array}{c}
0 \\
0 \\
1 \\
u_{1}-c_{1} \\
0
\end{array}\right), R^{(2)}=\left(\begin{array}{c}
1 \\
u_{2}-c_{2} \\
0 \\
0 \\
0
\end{array}\right), \\
R^{(4)}= & \left(\begin{array}{c}
u_{2}+c_{2} \\
0 \\
0 \\
0
\end{array}\right), R^{(5)}=\left(\begin{array}{c}
0 \\
1 \\
u_{1}+c_{1} \\
0
\end{array}\right), \\
R^{(3)}= & \left(\begin{array}{c}
\left(1-\gamma_{2}\right) p_{2}-\tilde{p} \\
c_{2}^{2}-\left(u_{2}-\tilde{u}\right)^{2} \\
\tilde{u} \frac{\left(1-\gamma_{2}\right) p_{2}-\tilde{p}}{c_{2}^{2}-\left(u_{2}-\tilde{u}\right)^{2}} \\
-\frac{\left(1-\gamma_{1}\right) p_{1}-\tilde{p}}{c_{1}^{2}-\left(u_{1}-\tilde{u}\right)^{2}} \\
-\tilde{u} \frac{\left(1-\gamma_{1}\right) p_{1}-\tilde{p}}{c_{1}^{2}-\left(u_{1}-\tilde{u}\right)^{2}} \\
1 \\
1
\end{array}\right) .
\end{aligned}
$$

The right eigenvectors for the primitive-variable formulation (12) are 


$$
\begin{aligned}
& R^{(1)}=\left(\begin{array}{c}
0 \\
0 \\
-\rho_{1} \\
c_{1} \\
0
\end{array}\right), R^{(2)}=\left(\begin{array}{c}
-\rho_{2} \\
c_{2} \\
0 \\
0 \\
0
\end{array}\right), R^{(3)}=\left(\begin{array}{c}
r_{1}^{(3)} \\
r_{2}^{(3)} \\
r_{3}^{(3)} \\
r_{4}^{(3)} \\
r_{5}^{(3)}
\end{array}\right), \\
& R^{(4)}=\left(\begin{array}{c}
\rho_{2} \\
c_{2} \\
0 \\
0 \\
0
\end{array}\right), R^{(5)}=\left(\begin{array}{c}
0 \\
0 \\
\rho_{1} \\
c_{1} \\
0
\end{array}\right),
\end{aligned}
$$

where $r_{1}^{(3)}=\frac{1}{\alpha_{2}} \frac{\tilde{p}-p_{2}+\rho_{2}\left(u_{2}-\tilde{u}\right)^{2}}{\left(u_{2}-\tilde{u}\right)^{2}-c_{2}^{2}}, r_{2}^{(3)}=\frac{1}{\alpha_{2} \rho_{2}}$ $\frac{\left(\tilde{u}-u_{2}\right)\left(\rho_{2} c_{2}^{2}-p_{2}+\tilde{p}\right)}{\left(u_{2}-\tilde{u}\right)^{2}-c_{2}^{2}}, r_{3}^{(3)}=-\frac{1}{\alpha_{1}} \quad \frac{\tilde{p}-p_{1}+\rho_{1}\left(u_{1}-\tilde{u}\right)^{2}}{\left(u_{1}-\tilde{u}\right)^{2}-c_{1}^{2}}$, $r_{4}^{(3)}=-\frac{1}{\alpha_{1} \rho_{1}} \frac{\left(\tilde{u}-u_{1}\right)\left(\rho_{1} c_{1}^{2}-p_{1}+\tilde{p}\right)}{\left(u_{1}-\tilde{u}\right)^{2}-c_{1}^{2}}$ and $r_{5}^{(3)}=1$.

It is easy to see that the characteristic fields $\lambda_{1}, \lambda_{2}, \lambda_{4}$ and $\lambda_{5}$ are genuinely nonlinear and the characteristic filed $\lambda_{3}$ is linearly degenerate for both the formulations.

\subsection{Riemann invariants}

Now, we introduce the linearly independent Riemann invariants corresponding to the $j$ th-characteristic field $(j=$ $1,2,4,5)$. The Riemann invariants corresponding to the characteristic field $\lambda_{1}$ is defined by

$$
\frac{d \rho_{2}}{0}=\frac{d u_{2}}{0}=\frac{d \rho_{1}}{-\rho_{1}}=\frac{d u_{1}}{c_{1}}=\frac{d \alpha_{1}}{0},
$$

and denote by $\Gamma_{k}^{1}, k=1, \ldots, 4$, i.e.,

$$
\Gamma_{1}^{1}=\rho_{2}, \Gamma_{2}^{1}=u_{2}, \Gamma_{3}^{1}=\frac{2 c_{1}}{\gamma_{1}-1}+u_{1}, \Gamma_{4}^{1}=\alpha_{1} .
$$

Similarly, for the characteristic fields $\lambda_{2}, \lambda_{4}$ and $\lambda_{5}$, the Riemann invariants are

$$
\begin{aligned}
& \Gamma_{1}^{2}=\frac{2 c_{2}}{\gamma_{2}-1}+u_{2}, \Gamma_{2}^{2}=\rho_{1}, \Gamma_{3}^{2}=u_{1}, \Gamma_{4}^{2}=\alpha_{1}, \\
& \Gamma_{1}^{4}=\frac{2 c_{2}}{\gamma_{2}-1}-u_{2}, \Gamma_{2}^{4}=\rho_{1}, \Gamma_{3}^{4}=u_{1}, \Gamma_{4}^{4}=\alpha_{1},
\end{aligned}
$$

and

$$
\Gamma_{1}^{5}=\rho_{2}, \Gamma_{2}^{5}=u_{2}, \Gamma_{3}^{5}=\frac{2 c_{1}}{\gamma_{1}-1}-u_{1}, \Gamma_{4}^{5}=\alpha_{1}
$$

respectively.
For the contact discontinuity associated with the characteristic field $\lambda_{3}=\tilde{u}$, we have

$$
\frac{d \rho_{2}}{r_{1}^{(3)}}=\frac{d u_{2}}{r_{2}^{(3)}}=\frac{d \rho_{1}}{r_{3}^{(3)}}=\frac{d u_{1}}{r_{4}^{(3)}}=\frac{d \alpha_{1}}{r_{5}^{(3)}},
$$

which imply the following relations

$$
\left.\begin{array}{l}
\Delta^{*} \rho_{2}=r_{1}^{(3)} \Delta \alpha_{1}, \\
\Delta^{*} u_{2}=r_{2}^{(3)} \Delta \alpha_{1}, \\
\Delta^{*} \rho_{1}=r_{3}^{(3)} \Delta \alpha_{1}, \\
\Delta^{*} u_{1}=r_{4}^{(3)} \Delta \alpha_{1},
\end{array}\right\}
$$

where $\Delta$ defined by $\Delta q=q_{R}-q_{L}$ and $\Delta^{*} q=q_{R}^{*}-q_{L}^{*}$.

\section{An approximate Riemann solver}

In this section, our purpose is now to construct an approximate Riemann solver for the system (14). We assume that all genuinely nonlinear characteristic fields are associated with rarefaction waves, we construct an approximate solution to the Riemann problem. This solution has closed form and is exact when, in addition, the jump in volume fractions is trivial.

\subsection{The four-rarefaction approximation}

Here we find an approximate solution by assuming that the four outer waves (nonlinear) are rarefaction waves. For this reason the approximation may be called a four-rarefaction Riemann solver and is an extension of the well-known tworarefaction approximation for compressible single phase gas dynamics. Obviously, if all nonlinear waves are in fact rarefactions, then the obtained solution is exact. Otherwise, our solution will be an approximation.

The approximate solution of the Riemann problem (14) has five waves, which are associated with the eigenvalues $\lambda_{1}=u_{1}-c_{1}, \lambda_{2}=u_{2}-c_{2}, \lambda_{3}=\tilde{u}, \lambda_{4}=u_{2}+c_{2}$ and $\lambda_{5}=$ $u_{1}+c_{1}$. The five waves are separated by six constant states. The middle wave is always a contact discontinuity corresponding to the eigenvalue $\lambda_{3}$ with the speed $\tilde{u}$, while the left and right waves are all rarefaction waves. Crucial in finding a solution to (14) is the determination of the two overlapping star regions either side of the contact, see figure 1 . The corresponding vectors $V_{L}^{*}$ and $V_{R}^{*}$ of unknowns are $V_{L}^{*}=\left(\rho_{2 L}^{*}, u_{2 L}^{*}, \rho_{1 L}^{*}, u_{1 L}^{*}, \alpha_{1 L}\right)^{t r}$ and $V_{R}^{*}=\left(\rho_{2 R}^{*}, u_{2 R}^{*}\right.$, $\left.\rho_{1 R}^{*}, u_{1 R}^{*}, \alpha_{1 R}\right)^{t r}$. Therefore, we have eight unknowns in the star region, since $\alpha_{1}$ only changes across the contact discontinuity.

Let $V_{L}$ and $V$ denote the left and the right states for a rarefaction wave. For a rarefaction wave, the two constant states $V_{L}$ and $V$ are connected through a smooth transition 


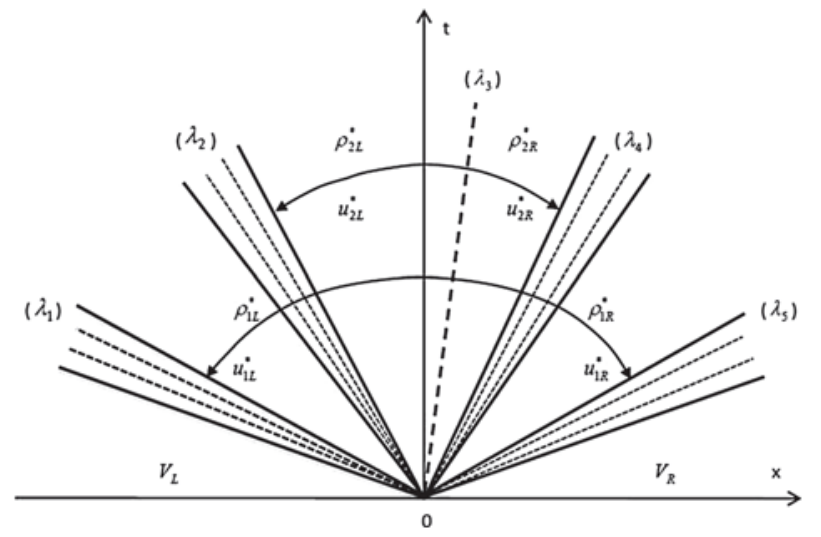

Figure 1. The Riemann problem structure on the $x-t$ plane for the one-dimensional time-dependent gas-liquid mixture twophase flow. The states $V_{L}$ and $V_{R}$ are given as input data to the problem. The genuinely nonlinear waves $\lambda_{1}, \lambda_{2}, \lambda_{4}$ and $\lambda_{5}$ that represent rarefaction waves with showing rarefaction fan and a linearly degenerate wave $\lambda_{3}$ that is a contact discontinuity.

in a $j$-th genuinely nonlinear characteristic field and the Riemann invariants are constant across the wave.

4.1a One-rarefaction wave for liquid phase: The Riemann invariants (18) are constant across one-rarefaction wave region, i.e., no change in density, velocity and volume fraction of gas phase and we have

$$
\frac{2 c_{1 L}^{*}}{\gamma_{1}-1}+u_{1 L}^{*}=\frac{2 c_{1 L}}{\gamma_{1}-1}+u_{1 L} \equiv \pi_{1 L},
$$

which imply

$$
u_{1 L}^{*}=\pi_{1 L}-\frac{2 c_{1 L}^{*}}{\gamma_{1}-1} .
$$

4.1b Two-rarefaction wave for gas phase: Across tworarefaction wave region, density, velocity and volume fraction of liquid phase are constant, therefore, we have

$$
u_{2 L}^{*}=\pi_{2 L}-\frac{2 c_{2 L}^{*}}{\gamma_{2}-1},
$$

where $\pi_{2 L}=\frac{2 c_{2 L}}{\gamma_{2}-1}+u_{2 L}$.

4.1c Four-rarefaction wave for gas phase: Again, the Riemann invariants (20) are constant for four-rarefaction wave region, i.e., $\Gamma_{1}^{4}$ is constant, therefore, we have

$$
\frac{2 c_{2 R}^{*}}{\gamma_{2}-1}-u_{2 R}^{*}=\frac{2 c_{2 R}}{\gamma_{2}-1}-u_{2 R} \equiv \pi_{2 R},
$$

which imply

$$
u_{2 R}^{*}=-\pi_{2 R}+\frac{2 c_{2 R}^{*}}{\gamma_{2}-1} .
$$

4.1d Five-rarefaction wave for liquid phase: For fiverarefaction wave region, $\Gamma_{3}^{5}$ is constant, therefore, we have

$$
u_{1 R}^{*}=-\pi_{1 R}+\frac{2 c_{1 R}^{*}}{\gamma_{1}-1},
$$

where $\pi_{1 R}=\frac{2 c_{1 R}}{\gamma_{1}-1}-u_{1 R}$.

Now we are going to prove two important theorems of a rarefaction wave for liquid phase.

Theorem 1 Across one-rarefaction wave (respectively, five-rarefaction wave), $\rho_{1}<\rho_{1 L}, u_{1}>u_{1 L}$ and $p_{1}<p_{1 L}$ (respectively, $\rho_{1}>\rho_{1 L}, u_{1}>u_{1 L}$ and $p_{1}>p_{1 L}$ ) if and only if, the characteristic speed increases from left hand to right hand state, i.e., $\quad \lambda_{1}\left(V_{L}\right)<\lambda_{1}(V) \quad$ (respectively, $\left.\lambda_{5}\left(V_{L}\right)<\lambda_{5}(V)\right)$, where $V_{L}$ and $V$ denote left and right states for rarefaction wave.

Proof Let us assume that for one-rarefaction wave, $\rho_{1}<\rho_{1 L}, u_{1}>u_{1 L}$ and $p_{1}<p_{1 L}$. Then we have to prove that the characteristic speed increases from the left to the right, that is, $\lambda_{1}\left(V_{L}\right)<\lambda_{1}(V)$. Using $c_{1}=\sqrt{d p_{1} / d \rho_{1}}$, we have $d c_{1} / d \rho_{1}>0, c_{1}$ is an increasing function of $\rho_{1}$; this implies that for one-rarefaction wave, $c\left(\rho_{1}\right) \leq c\left(\rho_{1 L}\right)$ or equivalently $-c_{1 L} \leq-c_{1}$. From $u_{1}>u_{1 L}$ and $-c_{1 L} \leq-c_{1}$ imply that $\lambda_{1}\left(V_{L}\right)<\lambda_{1}(V)$. In similar way, we can prove this for the five-rarefaction wave.

Conversely, let the characteristic speed increases from left hand to right hand state. Therefore from the inequality $\lambda_{1}\left(V_{L}\right)<\lambda_{1}(V)$ implies that

$$
c_{1}-c_{1 L}<u_{1}-u_{1 L},
$$

which by virtue of (23) becomes

$$
c_{1}+\frac{2 c_{1}}{\gamma_{1}-1}<c_{1 L}+\frac{2 c_{1 L}}{\gamma_{1}-1},
$$

which implies $\rho_{1}<\rho_{1 L}$ and $u_{1}>u_{1 L}$. Using $\rho_{1}<\rho_{1 L}$, we have $p_{1}<p_{1 L}$. Similarly, five-rarefaction wave, we can prove that $\rho_{1}>\rho_{1 L}, u_{1}>u_{1 L}$ and $p_{1}>p_{1 L}$.

Theorem 2 The one-rarefaction curve is convex and monotonic decreasing while five-rarefaction curve is concave and monotonic increasing.

Proof The one-rarefaction wave is

$$
u_{1}=\pi_{1 L}-\frac{2 c_{1}}{\gamma_{1}-1}, \text { if } \rho_{1}<\rho_{1 L},
$$

which on differentiation with respect to $\rho_{1}$, yields $\frac{d u_{1}}{d \rho_{1}}=$ $-\frac{c_{1}}{\rho_{1}}<0$, and subsequently,

$$
\frac{d^{2} u_{1}}{d\left(\rho_{1}\right)^{2}}=\frac{c_{1}}{\left(\rho_{1}\right)^{2}}-\frac{\left(c_{1}\right)^{\prime}}{\rho_{1}} .
$$



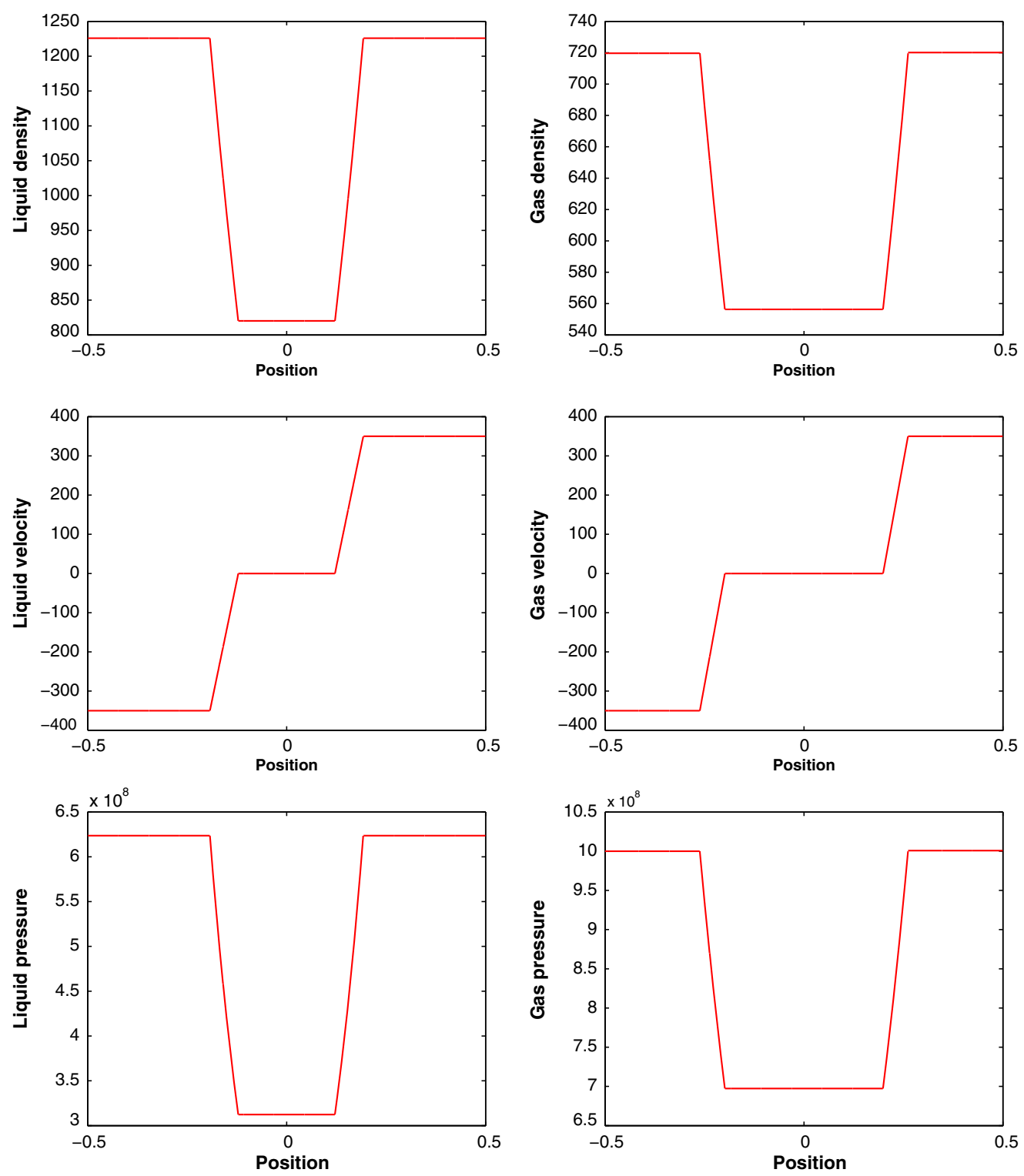

Figure 2. Test 1: The solution for density, velocity and pressure at time $t=1.5 \times 10^{-4}$ for both the phases with $K_{1}=$ $3.039 \times 10^{3}, K_{2}=1 \times 10^{5}, \gamma_{1}=1.72$ and $\gamma_{2}=1.4$. The exact solution consists of four symmetric rarefaction waves and a trivial contact discontinuity, that is $\alpha_{1 L}=\alpha_{1 R}$.

Using $p_{1}=K_{1} \rho_{1}^{\gamma_{1}}$ and $1<\gamma_{1}<2$, Eq. (28) yields $\frac{d^{2} u_{1}}{d\left(\rho_{1}\right)^{2}}>0$ and, therefore, $u_{1}$ is convex and monotonic decreasing with respect to $\rho_{1}$ for one-rarefaction waves. In a similar way, we can prove for five-rarefaction waves.

Similarly, it is easy to prove the following theorems of a rarefaction wave for gas phase.

Theorem 3 Across two-rarefaction wave (respectively, four-rarefaction wave), $\rho_{2}<\rho_{2 L}, u_{2}>u_{2 L}$ and $p_{2}<p_{2 L}$ (respectively, $\rho_{2}>\rho_{2 L}, u_{2}>u_{2 L}$ and $p_{2}>p_{2 L}$ ) if and only if, the characteristic speed increases from left hand to right hand state.
Theorem 4 The two-rarefaction curve is convex and monotonic decreasing while four-rarefaction curve is concave and monotonic increasing.

\section{Solution strategy}

The main physical quantities in the star region to be determined are $\rho_{2 L}^{*}, u_{2 L}^{*}, \rho_{1 L}^{*}, u_{1 L}^{*}, \rho_{2 R}^{*}, u_{2 R}^{*}, \rho_{1 R}^{*}$ and $u_{1 R}^{*}$. For each phase, we present a solution procedure to derive a system of two algebraic nonlinear equations for $\rho_{1 L}^{*}$ and $\rho_{2 L}^{*}$. 

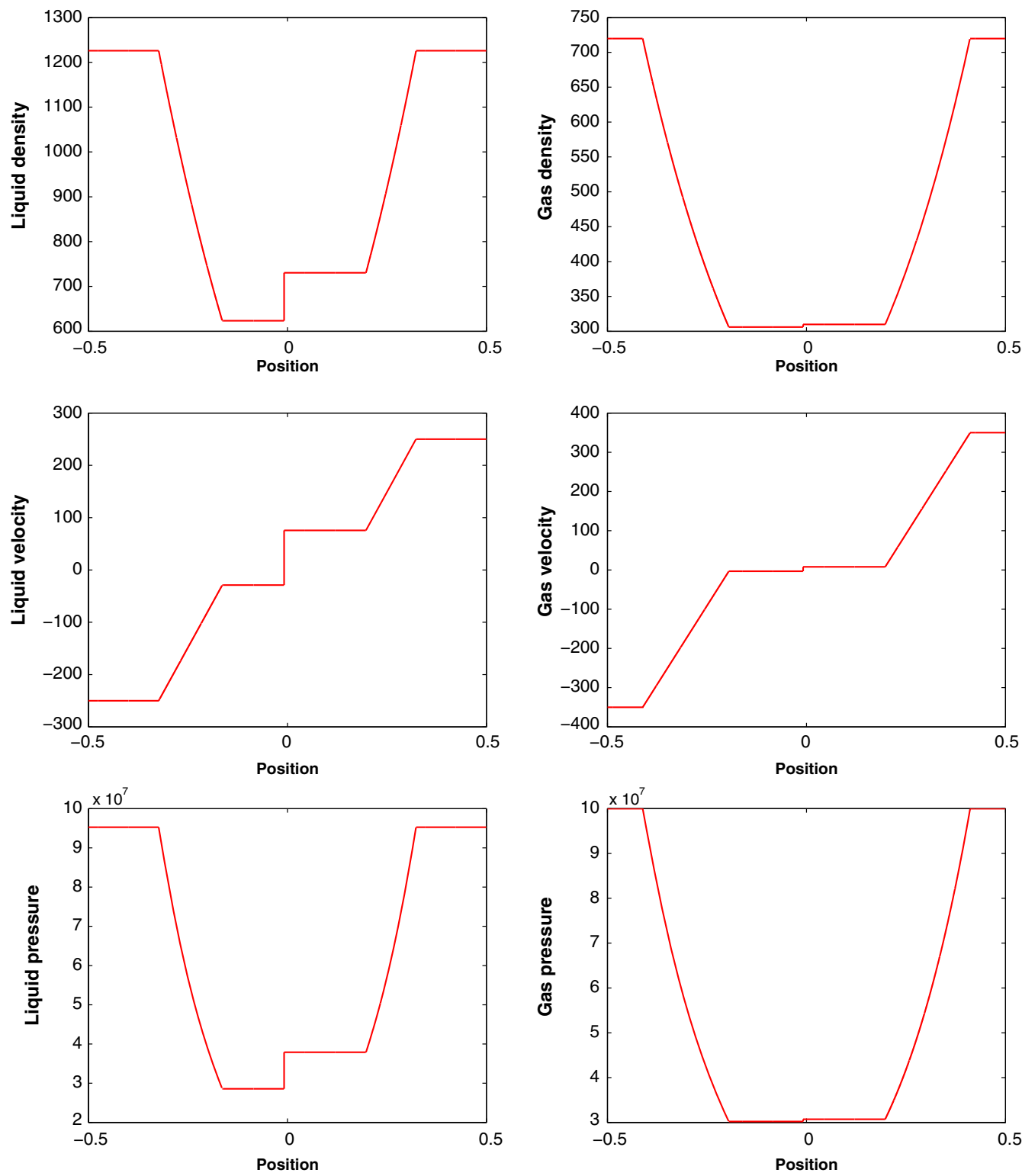

Figure 3. Test 2: The solution for density, velocity and pressure at time $t=5.2 \times 10^{-4}$ for both the phases with $K_{1}=303.0, K_{2}=$ $1.0 \times 10^{3}, \gamma_{1}=1.78$ and $\gamma_{2}=1.4$. The solution consists of four rarefaction waves and a non-trivial contact discontinuity, that is $\alpha_{1 L} \neq \alpha_{1 R}$.

Across the contact discontinuity associated with $\lambda_{3}=$ $\tilde{u}$ relations (22) hold. From the third and fourth relations of (22) and using (24), we obtain a single algebraic nonlinear equation for the unknown $\rho_{1 L}^{*}$ of the liquid phase is

$$
f\left(\rho_{1 L}^{*}, \rho_{2 L}^{*}\right)=0,
$$

where $\quad f=\eta_{1}\left[\left(\rho_{1 L}^{*}\right)^{\frac{\gamma_{1}-1}{2}}+\left(r_{3}^{(3)}\left(\alpha_{1 R}-\alpha_{1 L}\right)+\rho_{1 L}^{*}\right)^{\frac{\gamma_{1}-1}{2}}\right]-$ $\left(r_{4}^{(3)}\left(\alpha_{1 R}-\alpha_{1 L}\right)+\pi_{1 L}+\pi_{1 R}\right)$ and $\eta_{1}=\frac{2 \sqrt{K_{1} \gamma_{1}}}{\gamma_{1}-1}$.
Similarly, we have the single algebraic nonlinear equation for the unknown $\rho_{2 L}^{*}$ of the gas phase is

$$
g\left(\rho_{1 L}^{*}, \rho_{2 L}^{*}\right)=0,
$$

where $\quad g=\eta_{2}\left[\left(\rho_{2 L}^{*}\right)^{\frac{\gamma_{2}-1}{2}}+\left(r_{1}^{(3)}\left(\alpha_{1 R}-\alpha_{1 L}\right)+\rho_{2 L}^{*}\right)^{\frac{\gamma_{2}-1}{2}}\right]-$ $\left(r_{2}^{(3)}\left(\alpha_{1 R}-\alpha_{1 L}\right)+\pi_{2 L}+\pi_{2 R}\right)$ and $\eta_{2}=\frac{2 \sqrt{K_{2} \gamma_{2}}}{\gamma_{2}-1}$.

Now we consider the following two cases depending on the left and right initial data of the Riemann problem (14). 
Table 1. Initial data for trivial contact of the Riemann problem.

\begin{tabular}{cccccc}
\hline$V_{L}$ & $\rho_{2 L}$ & $u_{2 L}$ & $\rho_{1 L}$ & $u_{1 L}$ & $\alpha_{1 L}$ \\
\hline & 719.6856 & -350 & 1225.8912 & -350 & 0.9 \\
\hline$V_{R}$ & $\rho_{2 R}$ & $u_{2 R}$ & $\rho_{1 R}$ & $u_{1 R}$ & $\alpha_{1 R}$ \\
\hline & 719.6856 & 350 & 1225.8912 & 350 & 0.9 \\
\hline
\end{tabular}

Table 2. Initial data for non-trivial contact of the Riemann problem.

\begin{tabular}{cccccc}
\hline$V_{L}$ & $\rho_{2 L}$ & $u_{2 L}$ & $\rho_{1 L}$ & $u_{1 L}$ & $\alpha_{1 L}$ \\
\hline & 719.6856 & -350.0 & 1225.8912 & -250.0 & 0.1 \\
\hline
\end{tabular}

\begin{tabular}{cccccc}
$V_{R}$ & $\rho_{2 R}$ & $u_{2 R}$ & $\rho_{1 R}$ & $u_{1 R}$ & $\alpha_{1 R}$ \\
\hline & 719.6856 & 350.0 & 1225.8912 & 250.0 & 0.9 \\
\hline
\end{tabular}

Table 3. Initial data for Riemann problem.

\begin{tabular}{cccccc}
\hline$V_{L}$ & $\rho_{2 L}$ & $u_{2 L}$ & $\rho_{1 L}$ & $u_{1 L}$ & $\alpha_{1 L}$ \\
\hline & 719.6856 & 150 & 1225.8912 & 150 & 0.9 \\
\hline
\end{tabular}

\begin{tabular}{cccccc}
$V_{R}$ & $\rho_{2 R}$ & $u_{2 R}$ & $\rho_{1 R}$ & $u_{1 R}$ & $\alpha_{1 R}$ \\
\hline & 719.6856 & -150 & 1225.8912 & -150 & 0.9 \\
\hline
\end{tabular}

\subsection{Trivial contact}

If we consider the initial data like as $\alpha_{1 L}=\alpha_{1 R}$. Then using the condition $\alpha_{1 L}=\alpha_{1 R}$, from the Eqs. (29) and (30), we obtain the solution of the Riemann problem for both the phases as

$$
\left.\begin{array}{c}
\rho_{1 L}^{*}=\rho_{1 R}^{*}=\rho_{1}^{*}=\left(\frac{\pi_{1 R}+\pi_{1 L}}{2 \eta_{1}}\right) \frac{2}{\gamma_{1}-1}, \\
u_{1 L}^{*}=u_{1 R}^{*}=u_{1}^{*}=\frac{\pi_{1 R}-\pi_{1 L}}{2}, \\
\rho_{2 L}^{*}=\rho_{2 R}^{*}=\rho_{2}^{*}=\left(\frac{\pi_{2 R}+\pi_{2 L}}{2 \eta_{2}}\right) \frac{2}{\gamma_{2}-1}, \\
u_{2 L}^{*}=u_{2 R}^{*}=u_{2}^{*}=\frac{\pi_{2 R}-\pi_{2 L}}{2} .
\end{array}\right\}
$$

In this case there is no jump across the contact discontinuity and $V_{L}^{*}=V_{R}^{*}=\left(\rho_{2}^{*}, u_{2}^{*}, \rho_{1}^{*}, u_{1}^{*}, \alpha_{1}\right)^{t r}$, see figure 2. This is called trivial contact solution.

\subsection{Non-trivial contact}

For the general case $\alpha_{1 L} \neq \alpha_{1 R}$, there is a jump across the contact wave, see figure 3 . This is called non-trivial contact solution. The solution $V_{L}^{*}$ and $V_{R}^{*}$ is found by obtaining the roots of (29) and (30) numerically using Newton-Raphson method.

Assuming that we have computed $\rho_{1 L}^{*}$ and $\rho_{2 L}^{*}$ from (29) and (30), then we have the remaining solution for liquid phase

$$
\left.\begin{array}{c}
\rho_{1 R}^{*}=r_{3}^{(3)}\left(\alpha_{1 R}-\alpha_{1 L}\right)+\rho_{1 L}^{*}, \\
u_{1 L}^{*}=\pi_{1 L}-\eta_{1}\left(\rho_{1 L}^{*}\right) \frac{\gamma_{1}-1}{2}, \\
u_{1 R}^{*}=-\pi_{1 R}+\eta_{1}\left(\rho_{1 R}^{*}\right) \frac{\gamma_{1}-1}{2},
\end{array}\right\}
$$

and for gas phase

$$
\left.\begin{array}{c}
\rho_{2 R}^{*}=r_{1}^{(3)}\left(\alpha_{1 R}-\alpha_{1 L}\right)+\rho_{2 L}^{*}, \\
u_{2 L}^{*}=\pi_{2 L}-\eta_{2}\left(\rho_{2 L}^{*}\right) \frac{\gamma_{2}-1}{2}, \\
u_{2 R}^{*}=-\pi_{2 R}+\eta_{2}\left(\rho_{2 R}^{*}\right) \frac{\gamma_{2}-1}{2} .
\end{array}\right\}
$$

Note that in (32) and (33), $r_{1}^{(3)}$ and $r_{3}^{(3)}$ are the first and third components of the eigenvector $R^{(3)}$ depend on the unknowns of the problem. This leads us to various ways of solving the problem approximately.

We have been unable to find a direct solution for this case. We therefore introduce another level of approximation in order to obtain approximate values for $V_{L}^{*}$ and $V_{R}^{*}$ by solving iteratively, Eqs. (29) and (30). There are at least two ways of doing this.

(a) The simplest way is implemented by assuming that $r_{1}^{(3)}, r_{2}^{(3)}, r_{3}^{(3)}$ and $r_{4}^{(3)}$ in (29) and (30) are constant and are evaluated at the arithmetic means of their arguments using the initial conditions, that is $R^{(3)}=R^{(3)}(\hat{V})$, where $\hat{V}=$ $\left(V_{L}+V_{R}\right) / 2$. In this way the advantage is that the Eqs. (29) and (30) are in a single unknown variable $\rho_{1 L}^{*}$ and $\rho_{2 L}^{*}$, respectively. Therefore, these single variable equations are easy to handle for finding the roots using Newton-Raphson method.

(b) Now we assume that $r_{1}^{(3)}, r_{2}^{(3)}, r_{3}^{(3)}$ and $r_{4}^{(3)}$ in (29) and (30) are not constants. Then we have to solve the equations (29) and (30) which involve two unknown variables $\rho_{1 L}^{*}$ and $\rho_{2 L}^{*}$. Two unknown variables $\rho_{1 L}^{*}$ and $\rho_{2 L}^{*}$ in the star region can be found by solving the system of algebraic nonlinear equations (29) and (30). Here, we use the Newton-Raphson iterative procedure to find the roots of system of nonlinear algebraic equations in two variables.

\section{Numerical examples}

Densities $\rho_{1 L}^{*}$ and $\rho_{2 L}^{*}$ in the unknown region are found by solving the system of algebraic equations (29) and (30). Here, we use the Newton-Raphson iterative procedure to find the roots of these algebraic equations. In order to determine the solution inside a rarefaction wave for liquid phase, we proceed as follows. The left rarefaction wave is 

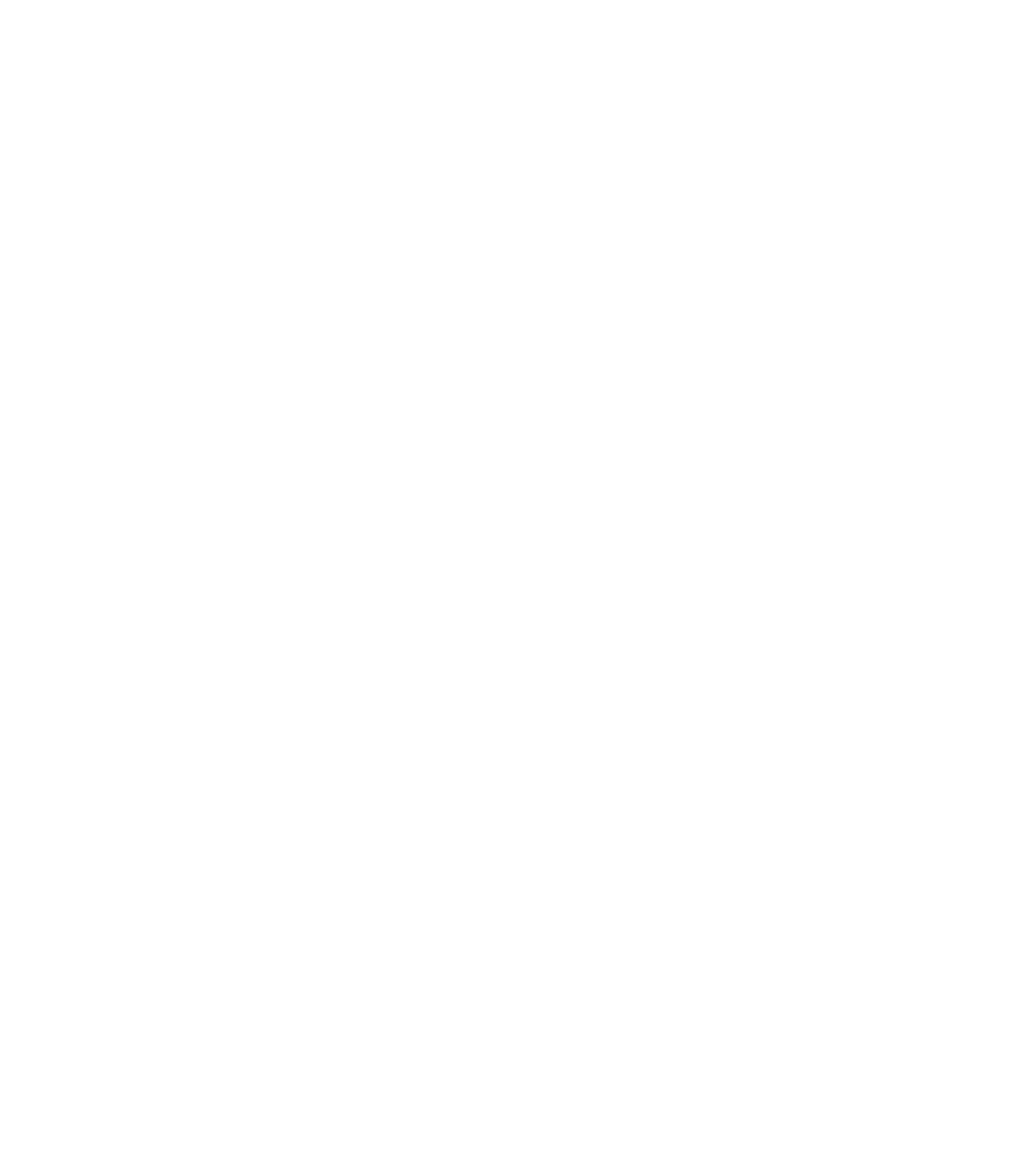

Figure 4. Test 3: The solution for density, velocity and pressure at time $t=1.2 \times 10^{-4}$ for both the phases with $K_{1}=$ $3.039 \times 10^{4}, K_{2}=1 \times 10^{5}, \gamma_{1}=1.72$ and $\gamma_{2}=1.4$. The solution consists of four symmetric shock waves and a trivial contact discontinuity, that is $\alpha_{1 L}=\alpha_{1 R}$.

enclosed by the head and tail characteristic wave fronts having speeds $s_{11}=u_{1 L}-c_{1 L}$ and $s_{12}=u_{1 L}^{*}-c_{1 L}^{*}$. It has been observed that the slope of a typical characteristic in the rarefaction wave region is

$$
\frac{d x}{d t}=\frac{x}{t}=u_{1}-c_{1}
$$

where $u_{1}$ and $c_{1}$ are to be determined at $(x, t)$. We obtain from (24) that

$$
\frac{x}{t}-\pi_{1 L}+\left(\frac{\gamma_{1}+1}{\gamma_{1}-1}\right) c_{1}=0
$$

which can be solved for $\rho_{1}$, where $\rho_{1 L}^{*}<\rho_{1}<\rho_{1 L}$ and subsequently we obtain $u_{1}$ inside the one-rarefaction wave. For the right rarefaction wave, the propagation of the speeds of the tail and head characteristic fronts are given by $s_{52}=u_{1 R}^{*}+c_{1 R}^{*}$ and $s_{51}=u_{1 R}+c_{1 R}$. In a manner similar to that followed for finding $\rho_{1} \in\left(\rho_{1 R}^{*}, \rho_{1 R}\right)$ by solving

$$
\frac{d x}{d t}=\frac{x}{t}=u_{1}+c_{1}
$$

and subsequently, we obtain $u_{1}$ inside the five-rarefaction wave. Similarly, we can obtain the solution inside a rarefaction wave for gas phase. Thus, the solution for the 
entire star region to the left and the right of the contact discontinuity is completely known.

The numerical examples presented for the cases of trivial and nontrivial contact discontinuities are from the test cases presented by Castro and Toro [11], for which the initial conditions are given in tables $1,2,3$. In test 1 the exact solution consists of four symmetric rarefaction waves and a trivial contact discontinuity, the solution profiles at time $t=1.5 \times 10^{-4}$ are shown in figure 2. For this case our theoretical solution is exact. In test 2 , the solution consists of four rarefaction waves and a non-trivial contact discontinuity; the solution profiles at time $t=5.2 \times 10^{-4}$ are shown in figure 3. For this example our theoretical solution is iterative and is only an approximation to the exact solution, which is unknown to us. Test 3 consists of four symmetric shock waves (weak) and a trivial contact discontinuity. For this case our theoretical solution can only be regarded as a crude approximation to the exact solution. First we remark that the theoretical correctness of shock wave solutions for non-conservative hyperbolic systems is currently unknown. Our theoretical approximation is based on the star values obtained from the four-rarefaction assumption and then we have estimated the shock speeds as an arithmetic mean of the characteristic speeds ahead and behind of the shock. See also figure 4 . From the solution of the Riemann problem, we illustrate some typical wave patterns using MATLAB. In the solution process, we choose to solve the model equations in a one dimensional domain of $[-0.5 ; 0.5]$.

\section{Conclusions}

We obtained the Riemann solution of a five-equation, twopressure (5E2P) model for compressible gas-liquid twophase flow with isentropic equations of state. The overall model is strictly hyperbolic and has no conservative form. We have developed an approximate solution, of complete wave structure of the Riemann problem with the assumption that waves associated with genuinely nonlinear characteristic fields are rarefaction, at any point $(x, t)$ in the relevant domain of interest $x_{L}<x<x_{R} ; t>0$, with $x_{L}<0$ and $x_{R}>0$. We have shown that the volume fractions change only across contact discontinuity and the solver is an exact for trivial contact. Numerical test cases are presented in the current paper and validate the current theoretical results for the simulation of the two-phase model. In future, we will extend this analytical procedure to construct the exact solution to the case of non-isentropic model of two phase flows.

\section{Acknowledgments}

Research support from the Human Resource Development (HRD) Group of Council of Scientific and Industrial Research
(CSIR), New Delhi, under the Senior Research Fellowship (SRF) scheme and Science and Engineering Research Board, Department of Science and Technology, Government of India (Ref No: SB/FTP/MS-047/2013) gratefully acknowledged by first and second authors respectively.

\section{References}

[1] Ransom V H and Scofield M 1976 Two-pressure hydrodynamic model for two-phase separated flow. Idaho National Engineering Laboratory SRD. pp 50-76

[2] Hicks D L 1981 Hyperbolic models for two-phase (or twomaterial) flow. NASA STI/Recon Technical Report No 82: 26624

[3] Ransom V H and Hicks D L 1984 Hyperbolic two-pressure models for two-phase flow. J. Comput. Phys. 53: 124-151

[4] Stewart H B and Wendroff B 1984 Two-phase flow: Models and methods. J. Comput. Phys. 56: 363-409

[5] Saurel R and Abgrall R 1999 A multiphase godunov method for compressible multifluid and multiphase flows. J. Comput. Phys. 150: 425-467

[6] Ishii M 1975 Thermo-fluid dynamic theory of two-phase flow. NASA STI/Recon Technical Report A 75: 29657

[7] Drew D 1983 Mathematical modeling of two-phase flow. Ann. Rev. Fluid Mech. 15: 261-291

[8] Andrianov N and Warnecke G 2004 The Riemann problem for the BaerBunziato two-phase flow model. J. Comput. Phys. 195: 434-464

[9] Schwendema D W, Wahle C W and Kapila A K 2006 The Riemann problem and a high-resolution Godunov method for a model of compressible two-phase flow. J. Comput. Phys. 212: 490-526

[10] Deledicque V and Papalexandris M V 2007 An exact Riemann solver for compressible two-phase flow models containing non-conservative products. J. Comput. Phys. 222: 217-245

[11] Castro C and Toro E F 2006 A Riemann solver and upwind methods for a two-phase flow model in non-conservative form. Int. J. Numer. Meth. Fluids 50: 275-307

[12] Murrone A and Guillard H 2005 A five equation reduced model for compressible two phase flow problems. J. Comput. Phys. 202: 664-698

[13] Baer M and Nunziato J 1986 A two-phase mixture theory for the deflagration-to-detonation transition (DDT) in reactive granular materials. Int. J. Multiphase Flow 12: 861-889

[14] Sharma V D 2010 Quasilinear hyperbolic systems, compressible flows, and waves. CRC Press, Boca Raton, FL

[15] Thanh M D 2014 A phase decomposition approach and the Riemann problem for a model of two-phase flows. J. Math. Anal. Appl. 418: 569-594

[16] Kuila S, Raja Sekhar T and Zeidan D 2015 A Robust and accurate Riemann solver for a compressible two-phase flow model. Appl. Math. Comput. 265: 681-695

[17] Kuila S, Raja Sekhar T and Zeidan D 2016 On the Riemann problem simulation for the drift-flux equations of two-phase flows. Int. J. Comput. Methods 13: 1650009

[18] Allaire G, Clerc S and Kokh S 2002 A five-equation model for the simulation of interfaces between compressible fluids. J. Comput. Phys. 181: 577-616 
[19] Raja Sekhar T and Sharma V D 2012 Solution to the Riemann problem in a one-dimensional magnetogasdynamic flow. Int. J. Comput. Math. 89: 200-216

[20] Radha R and Sharma V D 2012 Interaction of a weak discontinuity with elementary waves of Riemann problem. $J$. Math. Phys. 53: 013506

[21] Kuila S and Raja Sekhar T 2014 Riemann solution for ideal isentropic magnetogasdynamics. Meccanica 49: 2453-2465

[22] Kuila S and Raja Sekhar T 2016 Riemann solution for one dimensional non-ideal isentropic magnetogasdynamics. Comput. Appl. Math. 35: 119-133
[23] Ansari M and Ghiasi N 2012 Hydro dynamical instability initiation prediction by hyperbolic model for two-phase stratified flow using spectral method. Int. J. Comput. Methods Eng. Sci. Mech. 13: 1-9

[24] Toro E F 1999 Riemann solvers and numerical methods for fluid dynamics. Springer, New York 